management of labour and obstetric problems of immigrant women. There are also sections on the fetus and the newborn infant. The chapter on tropical obstetrics by Lawson is of great interest, discussing problems peculiar to these areas which are quite outside the experience of anyone who has worked only in western Europe and emphasizes the disadvantages under which medical and nursing staff have to work.

One great advantage of a textbook which has successfully survived so many editions is that techniques or methods of management which have largely been superseded are still included for reasons of completeness or comparison. This is particularly true of operations, in particular destructive operations on the fetus for, although rarely required, it is reassuring to be able to refer to these for the occasion when required.

This is an encyclopaedic, comprehensive and well written book which is more than adequate for the postgraduate student and a useful source of reference. The book under review is a low-priced edition published in soft-back form and, as such, represents excellent value at this price.

Prostate Cancer. A series of Workshops on the Biology of Human Cancer. Report No. 9 UICC Technical Report Series, vol. 48

Ed. by Donald S. Coffey and John T. IsaACs. Pp. 289, illustrated. International Union Against Cancer, Geneva, 1979. Sw. Fr. 20.00

Carcinoma of the prostate is the third commonest cause of death from cancer in the male and indeed evidence of carcinoma may be observed in something like one-third of prostates at post-mortem of men over 40 years of age, most of which, of course, are latent prostatic adenocarcinomas which grow very slowly or may never express clinical manifestations. It is not surprising, therefore, that there is an immense amount of both clinical and basic research engendered by this important topic, particularly since it is one of the few tumours that is hormonally dependent.

Prostate Cancer is the paper-back report, in typescript production, of a Workshop on prostatic cancer held in Geneva in October 1978 under the auspices of the International Union Against Cancer. It represents the deliberations of 12 experts, coming from the U.S.A., U.K., Belgium, Germany, Holland and Switzerland with specialities ranging over the fields of urology, endocrinology, pharmacology, biochemistry and pathology. The 15 chapters deal with the endocrine factors controlling normal prostatic growth and function, prostatic anatomy, the morbidity and mortality of prostatic cancer, animal models of the disease, treatment and therapeutic trials. There is an extensive discussion of the staging of prostatic cancer and methods of objective evaluation of response to therapy in an attempt to provide base lines for co-operative studies. Each chapter carries an extensive and up-to-date bibliography.

This is a volume that cancer specialists and urologists will turn to for up-to-date information on this important field. However, it is concentrated stuff and will not appeal to the casual reader!

\section{Recent Advances in Gastroenterology}

Edited by I. A. D. Bouchier. Vol. 4. Pp. ix +347 , illustrated. Churchill Livingstone, Edinburgh, London and New York, 1980. $£ 16.00$.

Consolidation of knowledge rather than conceptual innovations would seem to have characterized the last 4 years in the field of gastroenterology. In a series of thoroughly referenced articles, Professor Bouchier has drawn together contributions from Britain and America to cover 10 areas of current interest. These surveys touch on most aspects of gastrointestinal disease, taking account of the application of newer investigative techniques and advances in those basic sciences which relate to the gut. The latter approach is particularly illuminating in the section on the oesophagus which is now clearly shown to be integrated into the upper alimentary tract through neurological and hormonal mechanisms. The chapter on the stomach and duodenum, on the other hand, recognizes the paucity of recent advances of our understanding of diseases of this region, it concentrates instead on a most extensive review of progress in the medical and surgical treatment of peptic ulcer. The arguments as to whether to advise cimetidine intermittently at high dosage, semipermanently at lower dosage, or to advise elective surgery (proximal gastric vagotomy) for duodenal ulcer are thoroughly discussed in the light of recent trials. The colon is shown to be emerging from its obscurity at the nether regions of physiological research. Transit times, motility and bowe habit are, of course, revisited but absorption studies, shortchain fatty acid metabolism, microflora and gas production, and endocrine status are all seen to be growth areas. Disenchantment with carcino-embryonic antigen is justified, while the association between clostridial endotoxin and pseudomembranous colitis is accepted.

It is reassuring to note that the perennial problem of distinguishing ulcerative colitis from Crohn's disease still requires a measure of clinical experience. The aetiology of Crohn's disease remains unknown despite some attractive hypotheses. Experimental models for ulcerative colitis have implicated immune complexes and $\mathrm{T}$ cell mechanisms in pathogenesis. Treatment is evaluated and the importance of preventing growth retardation in children emphasized.

Hepatologists are well represented in 3 chapters. One pro vides a clear review of the complicated serology and epidemion ology of the hepatitis $A, B$, non-A and non-B viruses. $A$ further section covers diagnostic approaches to liver disorders, chronic active hepatitis, the treatment of hepatic encephalopathy, drugs (including alcohol) and the liver and liver tumours. In the third chapter, on the gall bladder and biliary tract, exciting changes are described, heralded by developments in radiology, ultrasound and endoscopy, and the expansion of knowledge concerning gall stone formation and dissolution using chenoseoxycholic acid.

The pancreas features in an analysis which specifically excludes the endocrine aspects of its function. The section concentrates most usefully on the morphological and functional investigation of pancreatic disorders. The theoretica validity of the traditional advice to 'rest' the pancreas in acute pancreatitis is questioned again; however, alternatives such as the use of protease inhibition or pharmacological resting agents (e.g. glucagon) remain unconvincing. No book of advances in this field would be complete without a comment on endoscopy, although it is the shortest review in the book, having already infiltrated many other sections. Its diagnostic role continues to expand with the use of transgastroscopic ultrasonography. Its developing therapeutic function is analysed particularly as a delivery system for various imaginative devices including lasers which inevitably attract attention. The book concludes with a review of aspects of paediatric gastroenterology, notably hepatic disorders, gastrointestinal protein intolerance and selective inborn errors of absorption.

In summary, this work is a mine of information, the style is readable and direct, the bibliography is extensive and the index adequate. Jt will be of use both to those working specifically in these fields and to the busy general physician wishing to browse through a well presented account of gastrointestinal subjects of current interest. 